\title{
Recommendation and Acceptance of Counselling for Familial Cancer Risk in Newly Diagnosed Breast Cancer Cases
}

\author{
Karin Kast ${ }^{a} \quad$ Julia Häfner ${ }^{b, c, d}$ Evelin Schröck ${ }^{c, d, e, f} \quad$ Arne Jahn ${ }^{c, d, e, f}$ \\ Carmen Werner $^{g}$ Cornelia Meisel $^{b, c, d}$ Pauline Wimberger $^{b, c, d}$ \\ ${ }^{a}$ Center of Familial Breast and Ovarian Cancer and Center of Integrated Oncology, University Hospital Cologne,

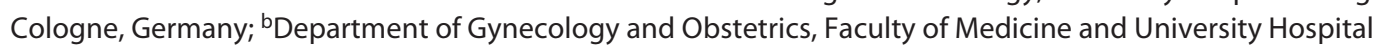 \\ Carl Gustav Carus, Technische Universität Dresden, Dresden, Germany; ${ }^{C}$ National Center for Tumor Diseases \\ (NCT), Partner Site Dresden, Dresden, Germany; ${ }^{\mathrm{d} G e r m a n}$ Cancer Consortium (DKTK) Dresden and German Cancer

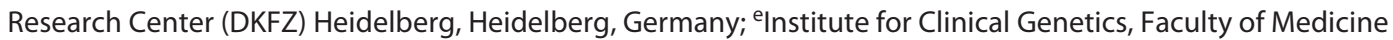 \\ and University Hospital Carl Gustav Carus, TU Dresden, Dresden, Germany; ${ }^{f}$ Hereditary Cancer Syndrome Center \\ Dresden, Faculty of Medicine and University Hospital Carl Gustav Carus, TU Dresden, Dresden, Germany; \\ ${ }^{9}$ Regional Clinical Cancer Registry, TU Dresden, Dresden, Germany
}

\section{Keywords}

Recommendation · Acceptance · Genetic · Counselling · Breast cancer

\begin{abstract}
Background: In clinical routine, not every patient who is offered genetic counselling and diagnostics in order to investigate a familial cancer risk predisposition opts for it. Little is known about acceptance of counselling and testing in newly diagnosed breast cancer cases in Germany. Methods: All primary breast cancer cases and patients with DCIS (ductal carcinoma in situ) treated at the University Hospital of Dresden between 2016 and 2019 were included. The number of tumor board recommendations for genetic counselling on the basis of the GC-HBOC risk criteria was recorded. Acceptance was analyzed by number of cases with counselling in the GC-HBOC-Center Dresden. Results: Of 996 primary breast cancer and DCIS cases, 262 (26.3\%) were eligible for genetic counselling. Recommendation for genetic counselling was accepted by $64.1 \%$ (168/262). Of these $90.5 \%$ (152/168) opted for molecular genetic analysis. The acceptance rate for counselling increased between 2016 and 2019 from 58.3 to $72.6 \%$. Altogether, $20.4 \%$ (31/152) patients were found to carry a pathogenic variant in the breast cancer
\end{abstract}

karger@karger.com www.karger.com/brc

Karger $\%$ BOPEN ACCESS
(C) 2021 The Author(s)

Published by S. Karger AG, Basel

This is an Open Access article licensed under the Creative Common Attribution-NonCommercial-4.0 International License (CC BY-NC) (http://www.karger.com/Services/OpenAccessLicense), applicable to the online version of the article only. Usage and distribution for commercial purposes requires written permission. genes BRCA1 or BRCA2. Conclusion: Acceptance of recommendation is increasing as clinical consequences augment. Optimization in providing information about hereditary cancer risk and in accessibility of counselling and testing is required to further improve acceptance of recommendation.

(c) 2021 The Author(s)

Published by S. Karger AG, Basel

\section{Introduction}

Only a fraction of patients who are offered counselling and molecular genetic diagnostics opt for it. Two US studies that explored the role of the surgeon found that the acceptance rate for genetic counselling after newly diagnosed breast cancer ranged between 59 and $78 \%$ depending on the surgeons attitude $[1,2]$. In other recommendation settings for breast and ovarian cancer patients, a much lower rate of $20.2 \%$ was described $[2,3]$. Importantly, most of the patients who present for genetic counselling subsequently undergo genetic analysis (7893\%) $[1,4]$. Although the genes BRCA1 and BRCA2 have been known for more than 2 decades, in many families with multiple cases of breast and ovarian cancers, a germline molecular genetic analysis has not been undertaken. At the same time, uptake of cascade testing in families 
Table 1. Characteristics of all primary breast cancer and DCIS cases

\begin{tabular}{|c|c|c|c|c|c|c|c|c|c|}
\hline & & \multirow{2}{*}{$\begin{array}{l}\text { Age at diagnosis, } \\
\text { years }\end{array}$} & \multicolumn{6}{|c|}{ Stage at primary diagnosis } & \multirow[t]{2}{*}{ Total } \\
\hline & & & 0 & I & II & III & IV & unknown & \\
\hline \multirow[t]{7}{*}{ Phenotype } & ER+, Her2-, G1/2 & $61.2[24-93]$ & 0 & 207 & 187 & 50 & 32 & 3 & $479(48)$ \\
\hline & ER+, Her2-, G3 & $54.4[27-90]$ & 0 & 31 & 66 & 18 & 9 & 1 & $125(13)$ \\
\hline & $\mathrm{ER}+, \mathrm{Her} 2+$ & $57.4[26-86]$ & 7 & 37 & 30 & 10 & 16 & 1 & $101(10)$ \\
\hline & ER-, Her2+ & $59.1[31-95]$ & 2 & 13 & 12 & 5 & 4 & 1 & $37(4)$ \\
\hline & ER-, Her2- & 58.5 [29-95] & 7 & 37 & 41 & 11 & 8 & 0 & $104(10)$ \\
\hline & Not known & $60.9[29-90]$ & 59 & 37 & 38 & 9 & 9 & 1 & $153(15)$ \\
\hline & Total & $59.51[24-95]$ & 75 & 359 & 374 & 103 & 78 & 7 & $996(100)$ \\
\hline \multirow[t]{3}{*}{ Chemotherapy } & Neoadjuvant* & $50.6[24-84]$ & 0 & 45 & 148 & 63 & 8 & 2 & 268 \\
\hline & Adjuvant & $59.2[32-82]$ & 0 & 41 & 66 & 16 & 4 & 0 & 127 \\
\hline & Both & $53.0[35-69]$ & 0 & 0 & 9 & 1 & 0 & 0 & 10 \\
\hline Hormonal therapy & & $61.0[24-92]$ & 13 & 189 & 185 & 44 & 38 & 2 & 469 \\
\hline Radiation & & $57.0[24-85]$ & 23 & 235 & 242 & 74 & 5 & 0 & 576 \\
\hline
\end{tabular}

Data are presented as mean [range], $N$, and $N(\%)$. ER, estrogen receptor; +, positive; -, negative; Her2, Her2neu receptor; G, grading. * Or primary systemic in stage IV breast cancer.

with a known pathogenic mutation is restrained [5-8]. It was reported to be $15-57 \%$ across 15 studies [5]. This is especially problematic where advanced stages of breast or ovarian cancer could have been prevented in very young women by participating in an intensive screening program or a prophylactic operation. In order to improve recommendation practice, implementation of the DKG (Deutsche Krebsgesellschaft, German Cancer Society) checklist and close cooperation with a department for clinical genetics is a requirement for certified breast cancer centers in Germany [3]. About 25\% of all cases who fulfil the risk criteria for familial breast and ovarian cancer will be carriers of a pathogenic variant in $B R C A 1$ or $B R C A 2$ [9]. Lifelong cancer risk in $B R C A 1 / 2$-carriers is $70 \%$ for breast and $20-45 \%$ for ovarian/tubal/peritoneal cancer [10]. Risk-reducing operations of the breast and primary subcutaneous mastectomy instead of breast conserving therapy is a point of consideration in carriers of $B R C A 1 / 2$ in patients with newly diagnosed breast cancer [11]. Moreover, better survival after risk-reducing salpingo-oophorectomy was described already in 2010 because of the high incidence of secondary cancer of ovaries or tubes in carriers [12]. Risk for contralateral breast cancer is especially high in young carriers with breast cancer. Participation in an intensified surveillance program or risk-reducing mastectomy is recommended depending on the prognosis of the primary breast cancer $[13,14]$. With olaparib and talazoparib, the first PARP inhibitors were approved for therapy in locally advanced or metastatic BRCA1/2-associated breast cancer in the EU in April 2019 and October 2020, respectively [15, 16]. But apart from individual recommendations for therapy and after care, the option of timely preventive measures for other family members who are carriers of a genetic predisposition very often is the primary reason for acceptance of genetic counselling and molecular genetic diagnostics in patients with newly diagnosed breast cancer [4]. The aim of this study was to investigate the acceptance of recommendation of genetic counselling in breast cancer cases in a University Hospital with a certified Breast Cancer Center, an accredited Department for Clinical Genetics and a Center for Hereditary Breast and Ovarian Cancer of the German Consortium (GC-HBOC).

\section{Methods}

All primary breast cancer cases and patients with DCIS (ductal cancer in situ) treated at the Department of Gynecology and Obstetrics at the University Hospital of Dresden were analysed. Early and advanced breast cancer cases are discussed in an interdisciplinary tumor board for therapeutic recommendations and for suspected hereditary cancer risk. The University Hospital Carl Gustav Carus Dresden hosts a certified breast cancer center and an accredited Department for Clinical Genetics, both of which together form one of the specialized centers of the GC-HBOC in Germany. The patient cohort consisted of all women with primary breast cancer or DCIS diagnosed between 2016 and 2019. Data on clinical stage (TNM and UICC classification), histopathological subtype, Her2-status, receptor status, and therapy were documented in the population-based Regional Cancer Registry Dresden (RKKRD). Data cut-off was in June 2020. Patients with a diagnosis of local breast cancer recurrence were excluded. Patients with prior molecular genetic analysis of the breast and ovarian cancer genes were excluded. Follow-up on acceptance of counselling and genetic testing was performed via alignment of the cases with patients who presented at the Center for Hereditary Breast and Ovarian Cancer (HBOC) in Dresden. All patients received interdisci- 


\section{Sporadic vs familial cases and BRCA-status of analyzed cases}

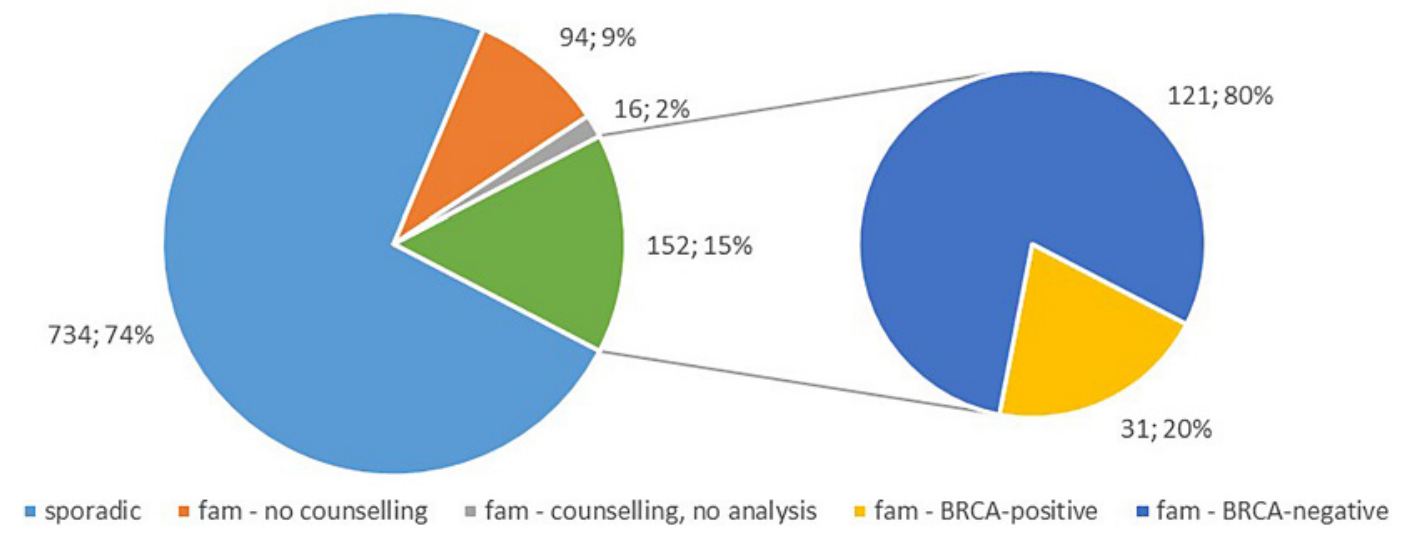

Fig. 1. Sporadic and hereditary cases of breast cancer or DCIS and germline BRCA status of eligible patients with genetic analysis.

plinary (human genetics/gynecology) counselling before molecular genetic diagnostics of the breast and ovarian cancer genes, mostly in a blood sample, was initiated (Panel sequencing and Array-based Comparative Genomic Hybridization). Pathogenic and likely pathogenic variants (class 4 or class 5 ) as well as variants of unknown significance (VUS, class 3) in the genes BRCA1 and $B R C A 2$ and in other breast and ovarian cancer predisposition genes were recorded [17]. However, only pathogenic and likely pathogenic variants in $B R C A 1 / 2$ were evaluated for the purpose of this study. Informed consent for further data analysis was obtained before documentation of the cases into the regional cancer registry and the HBOC database. The recommendations for or against further counselling based on the GC-HBOC hereditary cancer risk criteria were recorded for each case [18-20]. Acceptance was assessed by analysing the number of cases with interdisciplinary counselling in the GC-HBOC Center Dresden.

\section{Results}

From 2016 to 2019, the total number of cases of invasive breast cancer or DCIS at the University Hospital of Dresden was 996 . Of those, $92 \%$ were at early stage, and $8 \%$ presented with primary metastatic disease. Most of the breast cancers were hormone receptor positive, Her2 negative, grading 1 or 2 , representing the luminal A-subtype, and $10 \%$ were of the triple-negative subtype [21]. Of 477 invasive breast cancer cases with stage II or III, $61 \%$ were documented to have received chemotherapy in the neoadjuvant, adjuvant or in both settings. The characteristics of all breast cancer cases are displayed in Table 1 . $26.3 \%(262 / 996)$ of all patients fulfilled at least one GCHBOC hereditary cancer risk criteria triggering recommendation for interdisciplinary counselling and possibly
Table 2. Number of counselled patients $(n=168)$ with different GC-HBOC criteria of familial cancer risk and result of germline BRCA analysis

\begin{tabular}{llll} 
Criteria for BRCA analysis & Multiple criteria & \multicolumn{2}{l}{ BRCA positive } \\
\cline { 3 - 4 } & $(n=168)$ & $(n=31)$ & $\%$ \\
\hline $3 \geq \mathrm{BC}>51 \mathrm{y}$ & 44 & 11 & 26 \\
$2 \times \mathrm{BC}$, with $1 \times<51$ y & 106 & 25 & 24 \\
$\mathrm{BC}+\mathrm{OC}$ & 22 & 6 & 26 \\
$2 \times$ OC & 1 & 0 & 0 \\
$\mathrm{mBC}+\mathrm{BC}$ & 2 & 2 & 100 \\
$\mathrm{mBC}+$ OC & 0 & 0 & 0 \\
$\mathrm{BC}<36$ y & 41 & 16 & 40 \\
$\mathrm{bBC}<51$ y & 17 & 9 & 50 \\
$\mathrm{BC} / \mathrm{OC}$ & 7 & 2 & 29 \\
TNBC $<50$ y* & 33 & 13 & 41 \\
OC $<80 \mathrm{y}^{*}$ & 24 & 7 & 28 \\
Not specified, no analysis & 16 & 0 & 0 \\
\hline Total & 313 & 91 & 10 \\
\hline
\end{tabular}

$\mathrm{BC}$, breast cancer; $\mathrm{y}$, age at onset in years; $\mathrm{OC}$, ovarian cancer; $\mathrm{mBC}$, male breast cancer; bBC, bilateral breast cancer; TNBC, triple negative BC. * GC-HBOC criterion since January 2019.

genetic diagnostics (Fig. 1). Eligibility was mostly given by the risk criteria of a family with two women with breast cancer, one of them before the age of 51 years $(63 \%$; $106 / 168$ ). Of the 33 cases with triple negative breast cancer before the age of 50 years, 8 did not fulfill any of the other criteria. On average, each of the 168 patients with counselling recommendation fulfilled 1.9 of the GCHBOC criteria (Table 2). 
Fig. 2. Acceptance of recommendation of genetic counselling at the University Hospital Dresden (\% rounded to full numbers).

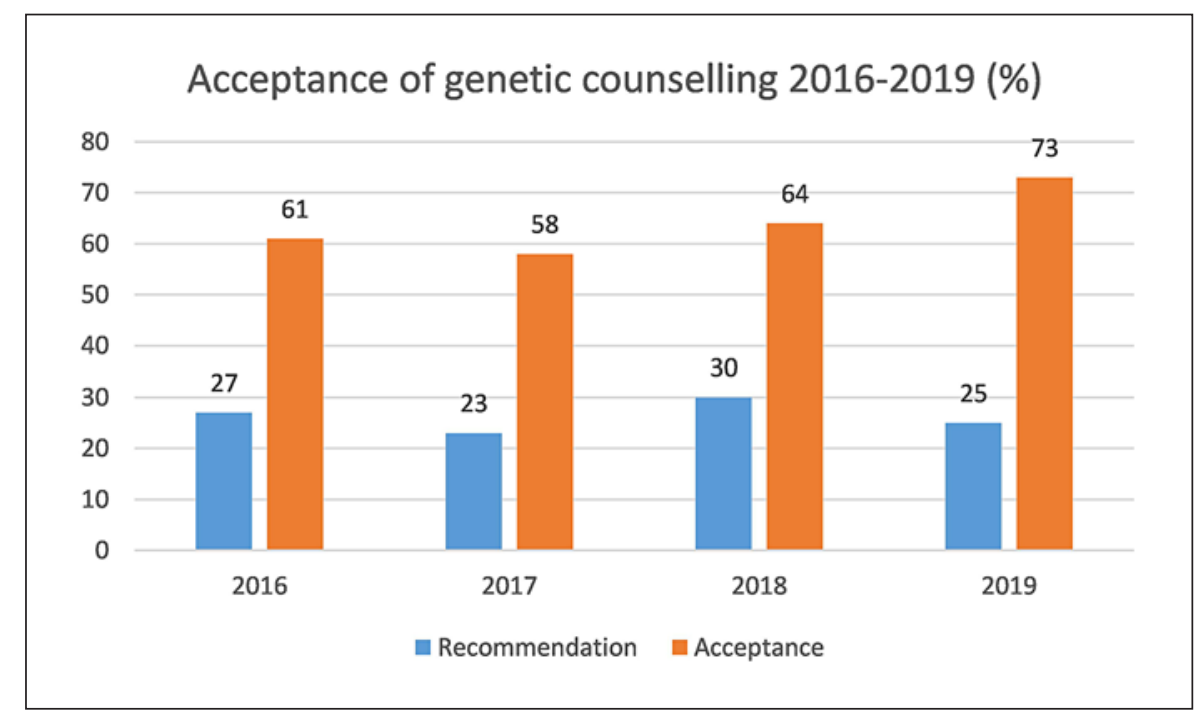

Acceptance of counselling was observed in $64.1 \%$ (168/262) of eligible patients. During the years 20162019 , acceptance of counselling for genetic cancer risk increased from 58.3 to $72.6 \%$, while recommendation for counselling was relatively steady and $26.3 \%$ on average (Fig. 2). Acceptance rate was slightly higher in cases who received neoadjuvant chemotherapy compared to no chemotherapy ( 74 vs. $67 \%$ ). After counselling, almost all patients opted for the comprehensive molecular genetic analysis $(90.5 \%$; 152/168) (Fig. 1).

Of the patients with genetic analysis, 20.3\% (31/152) were found to carry a pathogenic variant in the genes $B R C A 1$ or $B R C A 2$ (Fig. 1). Highest carrier rates were seen in the risk criteria that included male breast cancer or ovarian cancer (Table 2).

\section{Discussion}

In clinical routine, not every patient who is offered molecular genetic diagnostics in order to exclude a familial cancer risk predisposition opts for it. Little is known about acceptance of molecular genetic analysis in breast cancer cases in Germany. To evaluate the acceptance of counselling and molecular genetic analysis, all DCIS and breast cancer cases of the University Hospital Dresden between 2016-2019 were looked up for eligibility and having been counselled in the GC-HBOC center of the same hospital.

Altogether, $26.3 \%$ of the DCIS and breast cancer cases were eligible for germline analysis of the breast and ovarian cancer genes according to personal and family history after application of the DKG checklist and the additional criterion for single triple negative breast cancer. This is comparable with the results of a study of Rhiem et al. [3], where $30.4 \%$ of 5,091 probands of the region of Westphalia in Germany fulfilled the criteria for genetic testing according to the checklist. In our study, the individual probability for carrying a pathogenic variant was expectedly higher in families with additional cases of ovarian cancer or of male breast cancer [9].

In our study, $64.1 \%$ of patients who received counselling recommendation presented at the GC-HBOC Center of Dresden. This is a high acceptance rate compared to the literature. Rhiem et al.[3] described genetic testing of about $20 \%$ of the eligible patients in the Center of Cologne after 1 year of follow-up, whereas Kurian et al. [22] reported genetic testing of $52.9 \%$ of breast cancer patients with high risk for familial cancer 2 months after (surgical) operation. Interestingly, we observed a slightly higher acceptance rate for patients treated with neoadjuvant chemotherapy. During the neoadjuvant treatment, information about hereditary breast cancer risk and possible options for prevention might have been addressed more than once. In a questionnaire-based study, Scott et al. [23] acknowledge lack of information by clinicians as one of the reasons for low uptake of genetic testing. In the study of Rhiem et al. [3], the recommendation of genetic counselling was given by 10 local breast cancer centers. In contrast, in our study the information about eligibility and discussion of possible clinical consequences was provided by the same institution that offered the counselling. This underlines the importance of access to information of the breast surgeons about genetic testing, as it was shown to make a difference in the uptake of genetic counselling in the study of Katz et al. $[1,2]$. Interestingly, acceptance rate of genetic counselling in patients with ovarian cancer rose from $66 \%$ in 2013 to $>80 \%$ in 2015 after implementation of genetic counselling as a routine procedure in an oncologic clinic in close collabo- 
ration with a department for clinical genetics [24]. This clearly shows that information and optimal access improve utilization of healthcare services. Implementation of genetic counselling as part of the routine requirements for the treatment of breast and ovarian cancer should therefore be considered.

Within the 4 years, the rate of recommendation for counselling was relatively stable. The new criteria, offering counselling to women with TNBC before the age of 50 years since January 2019 might not have had a big impact as TNBC is a rare subtype [18]. Only $10 \%$ of all primary breast cancer cases are TNBC [25]. Additionally, most of them fulfil other criteria for counselling, and about $13 \%$ are diagnosed before the age of 36 years [26]. This might have been the reason for a similar recommendation rate in 2019 in spite of the additional GC-HBOC breast cancer risk criterion.

Importantly, the rate of acceptance of genetic counselling was slightly higher in $2019(72.6 \%)$. At the same time, the spectrum of clinical consequences enlarged, and methods of prevention became more and more feasible. An increasing number of germline BRCA1/2 variant carriers opt for risk-reducing mastectomies. This dynamic has most obviously been noted in the USA, but also, although to a lower degree, in Germany [27]. Currently, the BRCA germline status has limited consequences for medical treatment, but these can be of clinical benefit and support the relevance of genetic counselling and analysis for the affected individual. PARP inhibitor therapy was admitted for the treatment of advanced or metastatic BRCA1/2-associated breast cancer in April 2019 [15]. Since this study included exclusively cases of newly diagnosed breast cancer, no direct consequence for systemic therapy was given for this patient cohort. While the cohort size and the period of this study were relatively small, targeted therapeutic options could be certainly a reason for higher genetic testing acceptance in the future and should be discussed with the patients.

Although our acceptance rate is higher than others reported, it is still not satisfying from the medical point of view. The reasons for not presenting at the HBOC Center are the challenges of the current cancer therapy, no immediate clinical consequence and limited number of female family members [4]. Other more basic reasons are information deficits on the option for intensified surveillance and risk-reducing operations for affected carriers, cancer risk for male carriers and also discouragement by uninformed family members, doctors, or medical staff $[1,2]$. But also lack of education, access to medical treatment, language skills and insurance coverage were described as obstacles for acceptance of genetic counselling [4].

This study has potential limitations. We cannot exclude an ascertainment bias for patients going to an uni- versity hospital for treatment. In this case, a higher acceptance rate would be expected. Genetic diagnostics is not exclusively offered by the specialized HBOC Centers, but also by private institutes and doctor's offices. Acceptance might be higher than described by us, and some patients might have been counselled and tested elsewhere. Additionally information about genetic counselling and testing as well as RKKRD documentation might have been incomplete due to the data cut-off of 6 months for the last patients included. Most health insurance companies support the cooperation between GC-HBOC centers and local or regional breast and gynecologic cancer centers for routine counselling and testing of eligible patients since 2014 at the Center of Cologne and since 2019 at most of the other Centers (www.konsortium-familiaerer-brustkrebs.de). This setting might further increase the acceptance of the recommendation for genetic counselling at the GC-HBOC Center of Dresden within the next few years. But with regard to the initially mentioned obstacles in cascade testing, increased awareness and routine information of healthy women who see their gynecologic practitioner is also needed in the future.

In conclusion, we showed high acceptance of counselling and molecular genetic diagnostics for hereditary cancer risk in patients with newly diagnosed breast cancer. To reach as many individuals as possible, there is a need to further improve access to basic information and testing. Optimizing access to genetic counselling is key to taking advantage of preventive options in families at high risk for breast and ovarian cancer.

\section{Acknowledgements}

This work would not have been possible without the inspiration, concept, and continued effort of Rita Schmutzler, Christoph Engel, and all collaborators of GC-HBOC.

We thank the German Cancer Aid for supporting GC-HBOC with grant No. 110837.

We thank Antje Niedostatek from the Regional Clinical Cancer Registry Dresden (RKKRD) for data delivery and support.

\section{Statement of Ethics}

All procedures performed in studies involving human participants were in accordance with the ethical standards of the institutional research committee (No. EK 162072007) and with the 1964 Helsinki declaration and its later amendments or comparable ethical standards. This article does not contain any studies with animals performed by any of the authors. The study was approved by the ethics committee of the University Hospital of Dresden. Written informed consent was obtained from all individual participants included in the study. 


\section{Conflict of Interest Statement}

The authors declare that they have no conflict of interest.

\section{Funding Sources}

This work was supported by German Cancer Aid grant No. 110837 for GC-HBOC.

\section{Author Contributions}

K.K.: conception of the work, acquisition, analysis and interpretation of data, critical revision of the manuscript for important intellectual content, final approval of the version to be published.

J.H.: acquisition and analysis of data, final approval of the version to be published.

All other authors: acquisition of data, critical revision of the manuscript for important intellectual content, final approval of publication.

\section{References}

1 Katz SJ, Kurian AW, Morrow M. Treatment Decision Making and Genetic Testing for Breast Cancer: Mainstreaming Mutations. JAMA. 2015;314(10):997-8.

2 Hafertepen L, Pastorino A, Morman N, Snow J, Halaharvi D, Byrne L, et al. Barriers to genetic testing in newly diagnosed breast cancer patients: Do surgeons limit testing? Am J Surg. 2017;214(1):105-10.

3 Rhiem K, Bücker-Nott HJ, Hellmich M, Fischer H, Ataseven B, Dittmer-Grabowski C, et al. Benchmarking of a checklist for the identification of familial risk for breast and ovarian cancers in a prospective cohort. Breast J. 2019;25(3):455-60.

4 Bellcross CA, Peipins LA, McCarty FA, Rodriguez JL, Hawkins NA, Hensley Alford S, et al. Characteristics associated with genetic counseling referral and BRCA1/2 testing among women in a large integrated health system. Genet Med. 2015;17(1):43-50.

5 Menko FH, Ter Stege JA, van der Kolk LE, Jeanson KN, Schats W, Moha DA, et al. The uptake of presymptomatic genetic testing in hereditary breast-ovarian cancer and Lynch syndrome: a systematic review of the literature and implications for clinical practice. Fam Cancer. 2019;18(1):127-35.

6 Caswell-Jin JL, Zimmer AD, Stedden W, Kingham KE, Zhou AY, Kurian AW. Cascade Genetic Testing of Relatives for Hereditary Cancer Risk: Results of an Online Initiative. J Natl Cancer Inst. 2019;111(1):95-8.

7 Griffin NE, Buchanan TR, Smith SH, Leon AA, Meyer MF, Liu J, et al. Low rates of cascade genetic testing among families with hereditary gynecologic cancer: An opportunity to improve cancer prevention. Gynecol Oncol. 2020;156(1):140-6.

8 Bednar EM, Sun CC, McCurdy S, Vernon SW. Assessing relatives' readiness for hereditary cancer cascade genetic testing. Genet Med. 2020;22(4):719-26.

9 Kast K, Rhiem K, Wappenschmidt B, Hahnen E, Hauke J, Bluemcke B, et al. Prevalence of BRCA1/2 germline mutations in 21401 families with breast and ovarian cancer. J Med Genet. 2016;53(7):465-71.

10 Kuchenbaecker KB, Hopper JL, Barnes DR, Phillips KA, Mooij TM, Roos-Blom MJ, et al. Risks of Breast, Ovarian, and Contralateral
Breast Cancer for BRCA1 and BRCA2 Mutation Carriers. JAMA. 2017;317(23):2402-16.

11 Hartmann LC, Lindor NM. The Role of RiskReducing Surgery in Hereditary Breast and Ovarian Cancer. N Engl J Med. 2016;374(5): 454-68.

12 Domchek SM, Friebel TM, Singer CF, Evans DG, Lynch HT, Isaacs C, et al. Association of risk-reducing surgery in BRCA1 or BRCA2 mutation carriers with cancer risk and mortality. JAMA. 2010;304(9):967-75.

13 Rhiem K, Engel C, Graeser M, Zachariae S, Kast K, Kiechle M, et al. The risk of contralateral breast cancer in patients from BRCA1/2 negative high risk families as compared to patients from BRCA1 or BRCA2 positive families: a retrospective cohort study. Breast Cancer Res. 2012;14(6):R156.

14 Waha AVB, Kas t K, Kiechle M, Ditsch N, Meindl A, Niederacher D, et al. Konsensusempfehlung des Deutschen Konsortiums Familiärer Brust- und Eierstockkrebs zum Umgang mit Ergebnissen der Multigenanalyse. Geburtsh Frauenheilk. 2017;77(07): 733-9.

15 Robson ME, Tung N, Conte P, Im SA, Senkus $\mathrm{E}, \mathrm{Xu} \mathrm{B}$, et al. OlympiAD final overall survival and tolerability results: Olaparib versus chemotherapy treatment of physician's choice in patients with a germline BRCA mutation and HER2-negative metastatic breast cancer. Ann Oncol. 2019;30(4):558-66.

16 Litton JK, Hurvitz SA, Mina LA, Rugo HS, Lee $\mathrm{KH}$, Gonçalves A, et al. Talazoparib versus chemotherapy in patients with germline BRCA1/2-mutated HER2-negative advanced breast cancer: final overall survival results from the EMBRACA trial. Ann Oncol. 2020; 31(11):1526-35.

17 Wappenschmidt B, Hauke J, Faust U, Niederacher D, Wiesmüller L, Schmidt G, et al. Criteria of the German Consortium for Hereditary Breast and Ovarian Cancer for the Classification of Germline Sequence Variants in Risk Genes for Hereditary Breast and Ovarian Cancer. Geburtsh Frauenheilk. 2020;80(4): 410-29.

18 Engel C, Rhiem K, Hahnen E, Loibl S, Weber $\mathrm{KE}$, Seiler S, et al. Prevalence of pathogenic BRCA1/2 germline mutations among 802 women with unilateral triple-negative breast cancer without family cancer history. BMC Cancer. 2018;18(1):265.

19 Kast K, Rhiem K, Wappenschmidt B, Hahnen E, Hauke J, Bluemcke B, et al. Prevalence of BRCA $1 / 2$ germline mutations in 21401 families with breast and ovarian cancer. J Med Genet. 2016.

20 Harter P, Hauke J, Heitz F, Reuss A, Kommoss S, Marmé F, et al. Prevalence of deleterious germline variants in risk genes including BRCA $1 / 2$ in consecutive ovarian cancer patients (AGO-TR-1). PLoS One. 2017;12(10): $\mathrm{e} 0186043$

21 Goldhirsch A, Wood WC, Coates AS, Gelber RD, Thürlimann B, Senn HJ. Strategies for subtypes - dealing with the diversity of breast cancer: highlights of the St. Gallen International Expert Consensus on the Primary Therapy of Early Breast Cancer 2011. Ann Oncol. 2011;22(8):1736-47.

22 Kurian AW, Griffith KA, Hamilton AS, Ward KC, Morrow M, Katz SJ, et al. Genetic Testing and Counseling among Patients with Newly Diagnosed Breast Cancer. JAMA. 2017; 317(5):531-4.

23 Scott D, Friedman S, Telli ML, Kurian AW. Decision Making about Genetic Testing among Women With a Personal and Family History of Breast Cancer. JCO Oncol Pract. 2020;16(1):e37-55.

24 Rana HQ, Kipnis L, Hehir K, Cronin A, Jaung $\mathrm{T}$, Stokes SM, et al. Embedding a genetic counselor into oncology clinics improves testing rates and timeliness for women with ovarian cancer. Gynecol Oncol. 2021 Feb;160(2): 457-463.

25 Kast K, Link T, Friedrich K, Petzold A, Niedostatek A, Schoffer O, et al. Impact of breast cancer subtypes and patterns of metastasis on outcome. Breast Cancer Res Treat. 2015; 150(3):621-9

26 Shimelis H, LaDuca H, Hu C, Hart SN, Na J, Thomas A, et al. Triple-Negative Breast Cancer Risk Genes Identified by Multigene Hereditary Cancer Panel Testing. J Natl Cancer Inst. 2018;110(8):855-62.

27 Valero MG, Moo TA, Muhsen S, Zabor EC, Stempel M, Pusic A, et al. Use of bilateral prophylactic nipple-sparing mastectomy in patients with high risk of breast cancer. $\mathrm{Br} J$ Surg. 2020;107(10):1307-12. 